\title{
Reviews submitted from September 1st, 2015 to August 31st, 2016
}

\begin{tabular}{|c|c|c|c|}
\hline Carolyn Abitbol & Aysun Bayazit & Jesper Brok & Larry Cossey \\
\hline Khalad Abulaban & Laurence Beck & Ellen Brooks & Malcolm Coulthard \\
\hline Thurid Ahlenstiel & Rachel Becker-Cohen & Patrick Brophy & Richard Coward \\
\hline Rannar Airik & Brian Becknell & Elizabeth Brown & Jonathan Craig \\
\hline Ayse Akcan Arikan & Rolf Beetz & Alexandra Bruel & Brendan Crawford \\
\hline Abdullah Al Abbas & Nathan Beins & Timothy Bunchman & Erin Dahlinghaus \\
\hline R. Todd Alexander & Olga Beltcheva & Rainer Büscher & Murthy Darisipudi \\
\hline Uri Alon & Michael Bennett & Anja Büscher & John Daugirdas \\
\hline Harika Alpay & Shay Ben-Shachar & Lavjay Butani & Andrew Davenport \\
\hline Bruce Alpert & Albert Bensman & Necla Buyan & Miriam Davidovits \\
\hline Amira Al-Uzri & Etienne Bérard & Francois Cachat & Alan Davidson \\
\hline Sharon Andreoli & Kenrick Berend & Patrina Caldwell & Jean Claude Davin \\
\hline Bilal Aoun & Carsten Bergmann & Salim Caliskan & Liesbeth De Waele \\
\hline Mazen Arar & John Bertram & Cengiz Candan & Katherine Dell \\
\hline Klaus Arbeiter & Rajendra Bhimma & Francisco Cano & Claire Dempster \\
\hline Gianluigi Ardissino & Mario Bianchetti & Gabriel Cara-Fuentes & Michelle Denburg \\
\hline Gema Ariceta & Daniel Bichet & Thomas Carroll & Dev Desai \\
\hline Christina Arnold & O.N. Ray Bignall & Andrea Cassidy-Bushrow & Georges Deschênes \\
\hline Peter Aronson & John J. Bissler & J. Michael Cecka & Prasad Devarajan \\
\hline Elisabet Ars & Martin Bitzan & Ricard Cervera & Olivier Devuyst \\
\hline David Askenazi & Anna Bjerre & Vimal Chadha & Vikas R. Dharnidharka \\
\hline James Atherton & Petter Bjornstad & Deepa Chand & Jie Ding \\
\hline Meredith Atkinson & Markus Bleich & Alex Chang & Bradley Dixon \\
\hline Christoph Aufricht & Don Bliwise & Jennifer Charlton & Eiske Dorresteijn \\
\hline Ari Auron & Tom Blydt-Hansen & Swasti Chaturvedi & Jörg Dötsch \\
\hline Diego Aviles & Detlef Bockenhauer & Abanti Chaudhuri & Kevin Downes \\
\hline Arvind Bagga & Arend Bokenkamp & Arnaud Chiolero & Duska Dragun \\
\hline Sevcan A. Bakkaloglu & Melvin Bonilla-Felix & Min Hyun Cho & Laurence Dubourg \\
\hline Aysin Bakkaloglu & Dagmara Borzych-Dużałka & Mariann Churchwell & Jan Dudley \\
\hline Sushmita Banerjee & Maxime Bouchard & Mahmut Civilibal & Marina Easty \\
\hline Tom Barbour & Julie Boucquemont & Douglass Clayton & Aydin Ece \\
\hline Gina-Marie Barletta & Olivia Boyer & Joanne Clothier & Allison Eddy \\
\hline Biswanath Basu & Paul Brakeman & Pierre Cochat & Alberto Edefonti \\
\hline Rajit Basu & Michael C. Braun & Pilar Codoñer-Franch & Vidar Edvardsson \\
\hline Carl Bates & Eileen Brennan & Lawrence Copelovitch & Samir S. El-Dahr \\
\hline Don Batisky & Eileen Brewer & Rosanna Coppo & Ewa Elenberg \\
\hline Michel Baum & Joe Brierley & Marlies Cornelissen & Mohammed Elkhwad \\
\hline Harry Bawden & Luc P. Brion & Liane Correia-Costa & Francesco Emma \\
\hline
\end{tabular}




\begin{tabular}{|c|c|c|c|}
\hline Karlhans Endlich & Ian Griffin & Jennifer Jetton & Anne-Laure Lapeyraque \\
\hline Caroline Eng & Jaap W. Groothoff & Sally Johnson & Benjamin Laskin \\
\hline Elif Erkan & Marie-Claire Gubler & Rebecca Johnson & Christoffer Laustsen \\
\hline Christopher Esezobor & Luis Guerra & Pedro A. Jose & Moglie Le Quintrec-Donnette \\
\hline Robert Ettenger & Jean Pierre Guignard & Catherine Joseph & Sarah Ledermann \\
\hline Riccardo Faletti & Orlando Gutierrez & Shellie Josephs & Kathy Lee-Son \\
\hline Vassilios Fanos & Volker Haase & Shivani Joshi & Jens Leipziger \\
\hline Sahar Fathallah-Shaykh & Agnes Hackl & Janna Marie Journeycake & Mathieu Lemaire \\
\hline Janusz Feber & Dieter Haffner & Emily Joyce & Kevin Lemley \\
\hline Daniel Feig & Karsten Häffner & Philippe Jouvet & Sandrine Lemoine \\
\hline Guido Filler & Won-Ho Hahn & Bruce Julian & Rachel Lennon \\
\hline Richard N. Fine & David Hains & Shoji Kagami & Mary Leonard \\
\hline Gal Finer & George Hamilton & Jeffrey Kahn & Sandrine Leroy \\
\hline Michel Fischbach & Coral Hanevold & Koichi Kamei & Elena Levtchenko \\
\hline John Fleming & Jerome Harambat & Elaine S. Kamil & Christina Liakou \\
\hline Geoffrey Fleming & Pankaj Hari & Sylvia Kamphuis & Alexandre Liborio \\
\hline Frances Flinter & William Harmon & Yogavijayan Kandasamy & Christoph Licht \\
\hline Joseph T. Flynn & Erum Hartung & Kazunari Kaneko & Jean-Michel Liet \\
\hline Agnes Fogo & Asako Hayashi & Katsuyoshi Kanemoto & Fangming Lin \\
\hline John W. Foreman & Wesley Hayes & Bernard Kaplan & Beata Lipska-Ziętkiewicz \\
\hline Bethany Foster & James Heaf & Gaurav Kapur & Mieczyslaw Litwin \\
\hline Jean Francis & Laurence Heidet & Diana Karpman & Chantal Loirat \\
\hline Maria Franco & Lambertus van den Heuvel & Clifford Kashtan & David Long \\
\hline David Franz & Ian Hewitt & Frederick Kaskel & Michael Ludwig \\
\hline Veronique Fremeaux-Bacchi & Paul Hmiel & Lindsay Keir & Valerie Luyckx \\
\hline Aaron L. Friedman & Jacqueline Ho & Markus J. Kemper & John Mahan \\
\hline Yaacov Frishberg & Elisabeth Hodson & Alison Kent & Robert Mak \\
\hline Shuichiro Fujinaga & Johannes Hofer & Katarzyna Kiliś-Pstrusińska & Nizam Mamode \\
\hline Daniel Gale & Julien Hogan & Laura Klesse & Krissanapong Manotham \\
\hline Victor Garcia-Nieto & Ronald Hogg & Robert Kleta & Jianhua Mao \\
\hline Eduardo Garin & Christer Holmberg & Nine V.A.M. Knoers & Denise Marciano \\
\hline Arnaud Garnier & Gundela Holmdahl & Vera Koch & Loredana Marcovecchio \\
\hline Rouba Garro & Tuula Hölttä & Joshua Koch & Stephen Marks \\
\hline Jyothsna Gattineni & Masataka Honda & Paulo Koch Nogueira & Olivera Marsenic \\
\hline Rasheed Gbadegesin & Stephen R. Hooper & Amy Kogon & Shoichi Maruyama \\
\hline Simonetta Genovesi & Daljit Hothi & Valentina Kon & Michal Maternik \\
\hline Arlene Gerson & Alexander Howie & Martin Konrad & Robyn Matloff \\
\hline Gian Marco Ghiggeri & Peter Hoyer & Andreas Kortgen & Douglas Matsell \\
\hline Vasileios Giapros & Keith A. Hruska & Efstathios Koulouridis & Maria Chiara Matteucci \\
\hline Keisha Gibson & Kyu Huh & Larisa Kovacevic & Tej Mattoo \\
\hline Rodney Gilbert & Neil Hukriede & Rafael Kramann & Tim McCavit \\
\hline Charlotte Gimpel & Tracy Hunley & Jordan Kreidberg & Mignon McCulloch \\
\hline Mario Giordano & R. Morrison Hurley & Martin Kreuzer & Kirk McHugh \\
\hline Debbie Gipson & Kazumoto Iijima & Elaine $\mathrm{Ku}$ & Valerie McLin \\
\hline Richard Glassock & Yohei Ikezumi & Juhi Kumar & Stephen McWilliam \\
\hline Jens Goebel & Kenji Ishikura & Sachin Kumbhar & Mara Medeiros \\
\hline Aviva Goldberg & Khalid Ismaili & Christian Kurts & Otto Mehls \\
\hline Stuart Goldstein & Naomi Issler & Angela La Manna & Djalila Mekahli \\
\hline Jared Grantham & Shuichi Ito & Giuliana Lama & Francesca Mencarelli \\
\hline Marcella Greco & Micah Jacobs & Heather Lambert & Cathy Mendelsohn \\
\hline Melissa Gregory & Lyda Jadresic & Daniel Landau & Susan R. Mendley \\
\hline Gerald Greil & Timo Jahnukainen & Marc B. Lande & Shina Menon \\
\hline Ryszard Grenda & Hannu Jalanko & Craig Langman & Aicha Merouani \\
\hline
\end{tabular}


Timothy Meyer

Kevin Meyers

Maroun Mhanna

David Milford

Harald Mischak

Om Mishra

Mark Mitsnefes

Yosuke Miyashita

Modini Modem

Leo Monnens

Robert Montgomery

Giovanni Montini

Terry Moore

Michael L. Moritz

Marva Moxey-Mims

Dominik Müller

Luisa Murer

Imran Mushtaq

Michele Mussap

Koichi Nakanishi

Carla Nester

Galina Nesterova

Alicia Neu

Thomas Neuhaus

Thomas Nevins

Derek Ng

Stephanie Nguyen

Olivier Niel

Marina Noris

Victoria Norwood

Peter Nourse

Matti Nuutinen

Jun Oh

Gia Oh

Takayuki Okamoto

Eduardo Oliveira

Mario Ollero

Louise Oni

Rianne Oostenbrink

Edgar Otto

Leif Oxburgh

Fatih Ozaltin

April Padua

Fabio Paglialonga

Ulf Panzer

Lars Pape

Rulan Parekh

Dušan Paripović

Kwanjin Park

Paloma Parvex

Amira Peco-Antić

Elliot Perens

Licia Peruzzi
Mark Peters

Craig Peters

Catherine Peters

Stefano Picca

Amy Piepsz

Barbara Pieretti

Christine Pietrement

Martin Pohl

Anthony A. Portale

Hans Pottel

Madhura Pradhan

Manuel Praga

Narayan Prasad

Chanel Prestidge

Willem Proesmans

Marilynn Punaro

Uwe Querfeld

Raymond Quigley

Catherine Quinlan

Charles Quinn

Domonic Raj

Ali Ramezani

Patricio E. Ray

Lesley Rees

Jochen Reiser

Giuseppe Remuzzi

György S. Reusz

Michelle Rheault

Pornpimol Rianthavorn

Michael Riordan

Ken Roberts

Celia Rodd

Norman D. Rosenblum

Charles Rosenfeld

Caroline Rousset-Rouviere

David Rozansky

Zubaida Saifudeen

Khashayar Sakhaee

Alan Salama

Jeffrey Saland

Moin A. Saleem

Isidro B. Salusky

Matthew Sampson

Cheryl P. Sanchez

Simone Sanna-Cherchi

Fernando Santos

David Sas

Judy Savige

John Sayer

Asher Schachter

Jeffrey Schelling

Bruce Schlomer

Claus Peter Schmitt
H. William Schnaper

Frédérique Schortgen

Michiel Schreuder

Alan Schroeder

Andrew Schwaderer

George J. Schwartz

Neil J. Sebire

Tomas Seeman

Mouin Seikaly

David Selewski

Daniela Seracini

Sanjeev Sethi

Hannsjörg Seyberth

Pirouz Shamszad

Ibrahim Shatat

Vanessa Shaw

Ji-Nan Sheu

Masaki Shimizu

Jae Shin

Rukshana Shroff

Douglas M. Silverstein

Giacomo Simonetti

John Simpson

Sunder Sims-Lucas

Rakesh Sindhi

Aditi Sinha

Manish Sinha

Ekaterini Siomou

Roderick Skinner

Graham Smith

William Smoyer

Michael Somers

Brian Soriano

Inga Soveri

John Spencer

Vijay Srinivasan

Tarak Srivastava

Poyyapakkam Srivaths

Amy Staples

Constantinos Stefanidis

Karlijn van Stralen

José Strauss

Ram Sukhai

Kristina Suson

Scott Sutherland

Agnes Swiatecka-Urban

Jordan Symons

Tsukasa Takemura

Frederick Tam

Hiroshi Tanaka

Phillip I. Tarr

Gregory Tasian

Velibor Tasic
Jacob Taylor

Swasti Tiwari

E. Jane Tizard

Lidwien Tjaden

Matthew Tomey

Camilla Tøndel

Gabriele Tonni

Burkhard Tönshoff

Rezan Topaloglu

Kalman Tory

Howard Trachtman

Ha Tran

Jeng-Daw Tsai

Yong-Kwei Tsau

Shamir Tuchman

Kjell Tullus

Martin A. Turman

Katherine Twombley

Floris Udink ten Cate

Tim Ulinski

Maki Urushihara

Prayong Vachvanichsanong

Rudolph Valentini

Scott Van Why

Johan Vande Walle

Alex Vasilyev

Roberto Velasco

Priya Verghese

Enrico Verrina

Udo Vester

Enrico Vidal

Smitha Vidi

Bjorn Vikse

Marina Vivarelli

Konstantinos Voskarides,

Dean Wallace

Nicholas Ware

William Warzak

Toru Watanabe

Aoife Waters

Alan Watson

Donald Weaver

Lutz T. Weber

Stefanie Weber

Scott Wenderfer

Ulrich Wenzel

Katherine Wesseling-Perry

Oliver Wessely

Rik Westland

Derek Wheeler

Colin White

Delbert Wigfall

Jae van Wijk 
Lore Willem

Matthias Wolf

William Wong

Craig Wong

Adrian Woolf

Anne Wright

Elke Wühl

Robert J. Wyatt

Fatos Yalcinkaya

Wanling Yang
Hui-Kim Yap

Elisa Ylinen

Norishige Yoshikawa

Ihor Yosypiv

Zihua Yu

Ariane Zaloszyc

Alberto Zanchetti

Michael Zappitelli

Joshua Zaritsky

Israel Zelikovic
Yongdong Zhao

Gaston Zilleruelo

Peter Zipfel

Aleksandra Zurowska

Danuta Zwolinska 